Loll, F., Scheuer, O., McLaren, B.M.,\& Pinkwart, N. (2010). How can students learn to argue from computer-based systems? A survey of teachers, researchers, and system developers. In M. Wolpers, P. A. Kirschner, M. Scheffel, S. Lindstaedt, \& V. Dimitrova (Eds.), Proceedings of the 5th European Conference on Technology Enhanced Learning, Sustaining TEL: From Innovation to Learning and Practice (EC-TEL 2010), LNCS 6383, September/October 2010, Barcelona, Spain. (pp. 530-535).

\title{
Computer-Supported Argumentation Learning: A Survey of Teachers, Researchers, and System Developers
}

\author{
Frank Loll ${ }^{1}$, Oliver Scheuer ${ }^{2}$, Bruce M. McLaren ${ }^{2}$, Niels Pinkwart ${ }^{1}$ \\ ${ }^{1}$ Clausthal University of Technology, Department of Informatics, \\ \{frank.loll, niels.pinkwart\}@tu-clausthal.de \\ ${ }^{2}$ German Research Center for Artificial Intelligence (DFKI), \\ \{oliver.scheuer, bmclaren\}@dfki.de
}

\begin{abstract}
Argumentation is omnipresent in our lives and therefore an important skill to learn. While classic face-to-face argumentation and debate has advantages in helping people learn to argue better, it does not scale up, limited by teacher time and availability. Computer-supported argumentation (CSA) is a viable alternative in learning to argue, currently increasing in popularity. In this paper, we present results from a survey we conducted with experts on argumentation learning systems, one which provides a glimpse on future directions.
\end{abstract}

Keywords: Argumentation, Survey, CSCL

\section{Introduction}

Argumentation is omnipresent in our lives. Nevertheless, people often struggle to engage in reasoned arguments [1], making the acquisition of argumentation skills an important educational goal. Traditional face-to-face teaching methods are limited by teacher time and availability. To remedy these limitations, researchers have developed computer-based systems to facilitate the acquisition of argumentation skills.

In a detailed review of over 50 argumentation systems and methods, we surveyed the current state of the art for educational argumentation tools [2]. In addition, to better understand the decisions that have influenced the design of such systems, and to inquire into the most promising current developments and future trends, we recently conducted a web-based survey among argumentation teachers, researchers and system developers. The survey comprised four parts. First, participants were informed about the purpose of the survey, the use of the data and whether they wanted to receive the anonymized results once the analysis was completed. Second, the participants were asked about their professional background in the area of argumentation. All participants with a high degree of self-reported experience in at least one area of research, teaching and designing/developing of argumentation systems were, in the third part, asked specific multiple-choice questions about the research questions listed in section 2 below. Finally, the participants were prompted for free-text responses to open-ended questions. 
The survey participants are experts in argumentation (research, teaching and developing technology). To select these experts, we collected a list of about 40 persons who we knew to have expertise through our research on the above-mentioned review article [2]. In a second step, we systematically searched through the author lists of relevant conferences (ITS, AIED, CSCL) and journals (ijCSCL) and carried out an exploratory Google search, checking home pages and publications lists, resulting in an extended list of 153 experts. We then invited the experts via e-mail to take part in the web-based survey. Participation in the survey was voluntary. As motivation we raffled an Apple iPod among all participants. In total, we received 97 responses in the (approximately) two months the questionnaire was online.

In [3] we discuss the quantitative findings of this survey. Here, we enrich these results with an analysis of the participants' free text responses.

\section{$2 \quad$ Research Questions}

In the survey, we were interested in the following research questions (RQ):

(1) Are visual argument representations helpful for learning and/or understanding argumentation?

(2) Can computer-supported / computer-mediated argumentation replace face-to-face argumentation?

(3) Does the formality of a domain influence the type of collaboration that is appropriate?

(4) Do argumentation researchers, teachers and system developers differ in their views on the suitability of collaboration for argument learning?

(5) Is it possible to develop automated analysis features that can effectively analyze arguments?

(6) Are there domain-specific differences that influence the suitability of automated analyses?

(7) How and when is tutorial feedback most effectively provided?

\section{Results and Discussion}

Our discussion is structured along three major themes, which map to the above research questions as follows: visual representation of arguments (1), individual vs. collaborative argumentation (2-4), and analysis and feedback (5-7).

\subsection{Category 1: Visual Representation of Arguments}

Overall, in the quantitative questions, there was a strong agreement across different domains (e.g., the law, science), that visual representations of arguments help people to gain an understanding of the topic of the argumentation (RQ 1) [3]. We asked the survey question "Imagine that you have a software tool with graphical components 
representing different plausible argument moves that users can choose from. They might be able to choose from components such as claim, fact, or rebuttal and then fill in the selected shapes with text specific to their idea. Do you think such an approach would help or hinder users as they construct arguments and why?" The positive responses to the question included reasons such as:

- Graphical representations help organize one's thoughts (e.g., "it can facilitate the overall process in many ways, such as ... in maintaining focus on the overall process... maintaining consistency and in increasing plausibility and accuracy")

- They serve as an external memory aid

- They are a good support for collaborative activities

- Visually represented arguments prevent biases (e.g., "students (...) cognitive biases prevent them from making good arguments. Diagrams can help overcome these limitations by (...) converting a memory-based cognitive operation into a more formal visual operation")

On the other hand, some respondents also argued against visually represented argument structures:

- Visual representations have limited expressiveness (e.g., "research shows they feel too constricted by such systems.")

- Visualizing arguments is artificial and only applicable to simple arguments

- Visualization may get in the way (e.g., "experienced learners may be hindered to apply their advanced strategies")

- Additional visual representations may increase cognitive load

\subsection{Category 2: Individual vs. Collaborative Argumentation}

As our prior review revealed, one of the key differences between existing argumentation systems is the support for collaboration. In our survey, most experts agreed on the role of computers to support groups of people in conducting useful, valid arguments. However, the agreement dropped when the experts were asked if computers could replace face-to-face argumentation among learners (RQ 2). In addition, there was a noteworthy correlation between the formality of arguments and the role of individual learning among experts in educational argumentation (RQ 3).

In a free-text question, we asked "Can you describe the typical process and roles that are used in arguments (or debates between parties) in your primary domain of interest?" Here, the responses indicated that most arguments are multi-party and that argument processes are complex, involving multiple phases (e.g., preparation, engagement with clarifications, countering arguments, solution suggestions, decisions and modifications). Also, a variety of group learning modes were suggested, including:

- One participant taking a position and defending it against the others (e.g., "students are asked to take a position regarding the controversy, to support their positions with reasons.")

- Rotating roles

- Criticizing and modifying positions in groups 
- Formation of different parties with different perspectives on the argument (e.g., "there would be a whole-class discussion where students present both sides of the debate."

The survey also included a question asking "What kinds of problems occur most frequently when your students practice argumentation collaboratively with one another? (As opposed to composing arguments on their own)?" Here, responses varied. Some key problems repeatedly mentioned were:

- Students biased towards their own viewpoint (e.g., "they tend to argue by supporting their own standpoint/claim without reflecting on the connection between their standpoint/claim and the opponent's one")

- Agreement or disagreement problems, in particular, students agreeing too easily (e.g., "Typically, I see students who tend to agree with one another and hesitate to disagree with someone's point of view.")

- Student having trouble with collaborative argumentation due to a lack of argumentation skills

\subsection{Category 3: Feedback Techniques}

Imbuing argumentation systems with automated tutoring and feedback functionality holds promise to increase learning by adapting to individuals, groups, processes and situations. An important prerequisite for providing tutorial feedback is the ability to analyze and understand students' arguments to a sufficient extent, i.e. to identify weaknesses and to assess the quality of students' arguments in order to inform appropriate feedback. Due to the open-textured nature of arguments the development of effective analysis mechanisms is non-trivial, however. In the survey, we asked the question "In your primary domain of interest, what are the most common mistakes made by students (or typical misconceptions) in formulating arguments on their own (i.e., individually)?" Here, the responses show two main areas: arguments that are weak in form and structure, and problems with evidence. Related to the first area, the respondents' comments included remarks concerning

- Student's argument logic being weak overall (e.g., "so they listed several possible arguments without real explanations or they preferred to focus on one argument only and try to articulate different aspects of this unique argument, they lacked in this case coordinating several arguments on a same topic to answer a controversial question.")

- Student's failure to see overall, recurring patterns in arguments

- Problems with argument structures (e.g., "Misconception of node/link ontology")

Related to the second area (evidence), the main problems of students (as reported on by our respondents) were

- Not recognizing the difference between evidence and claims (e.g., "A lot of students can't distinguish between claim and argument.")

- Not providing (enough) supporting evidence 
- Not recognizing important evidence (e.g., "Students rely too much on intuition and they work to defend their current beliefs, despite evidence and arguments to the contrary.")

- Accepting false evidence

To what extent is the development of effective analysis mechanisms feasible (RQ $5)$ ? Our experts showed a tendency to believe in the existence of general and recurring patterns that indicate errors and weaknesses in their domain of interest, a tendency that increases with the amount of teaching experience [3]. This finding is not surprising because it is part of a teacher's job to identify such patterns of errors and weaknesses. There was less confidence in the feasibility of defining sets of rules to automatically identify these patterns, and even less confidence in the feasibility of automated assessment of argument quality. The difficulties inherent in assessments of arguments are also clear from current and past argumentation learning systems: Tutorial support is often based on explicitly structured argument representations (e.g., argument graphs in Belvedere [4] and LARGO [5]) rather than on the more difficult task of assessing textual content. An important variable that might bear on the feasibility of automated analysis is the specific domain of interest, e.g., more formal domains might be easier to analyze due to their explicit elements and structure (RQ 6). This assumption, however, was not confirmed. Only one (statistically nonsignificant) tendency was identified with respect to pattern-based quality assessment. Experts from the legal domain exhibited the most skeptical stance. Interestingly, legal experts also assessed their knowledge in artificial intelligence techniques significantly higher than experts in other domains, i.e. they might have been the most aware of the difficulties of an automated quality assessment.

Another important question is when and how to react to students' errors and misconceptions (RQ 7). Obviously, the answer to the question how to react depends strongly on the specific domain, student knowledge level, and instructional goals. In the survey, we asked "What general types of feedback do you (or would you) give to students when they make oral or written arguments in your primary domain of interest?" Here, the answers indicate a wide spectrum of possible teacher interventions, including

- "asking questions."

- $\quad$ "I would also ask student to consider the best arguments supporting alternative views."

- "I try to give them feedback that reinforces the idea that there are general principles of argument that they must strive to respect."

- 'Individually, then, we provide careful correction of each student's analysis."

- " the feedback given to students is ... 'expert/human' when a learning partner reflects upon the arguments."

- "I might use one or more of the models of argument to draw their attention to elements that are missing or poorly connected."

- "Most of the feedback would be in the forms of challenges e.g ... how might somebody argue against you?" 


\section{Conclusion}

In this paper, we summarized the results of data collected as part of a web-based survey among experts from argumentation research, argumentation teaching and argumentation system development, particularly focusing on examples from free-text questions (cf. [3] for the quantitative data analysis). There was considerable agreement that argumentation systems are able to facilitate learning via argument visualization techniques; on the other hand, some respondents argued against graphical argument visualization. In addition, the experts agreed that computers have proven their suitability in promoting collaborative learning of argumentation. Yet, some dangers and risks of CSCL practices for argumentation learning were also mentioned, such as that students tend to agree too easily and thus not learn much. A still open issue is the future and application potential of computer-based analysis and feedback on argumentation. Here, many respondents see great potential; on the other hand, there was no agreed-upon "ideal" form of (even human) argument analysis and feedback. This points to the clear challenges that face those working on automated, computer based analysis and feedback.

\section{Acknowledgments}

We thank all respondents and pilot testers of the questionnaire. This work was supported by the German Research Foundation (DFG) under the grant "LASAD".

\section{References}

1. Kuhn, D.: The skills of argument. Cambridge: Cambridge University Press (1991)

2. Scheuer, O., Loll, F., Pinkwart, N., McLaren, B. M.: Computer-Supported Argumentation: A review of the state of the art. International Journal of Computer-Supported Collaborative Learning, 5(1), 43-102 (2010).

3. Loll, F., Scheuer, O., McLaren, B. M., Pinkwart, N.: Learning to Argue Using Computers A View from Teachers, Researchers, and System Developers. In Proceedings of ITS2010, Part II, LNCS 6095, pp. 377-379 (2010).

4. Suthers, D. D., Connelly, J., Lesgold, A., Paolucci, M., Toth, E. E., Toth, J., Weiner, A.: Representational and advisory guidance for students learning scientific inquiry. In K. D. Forbus \& P. J. Feltovich (Eds.), Smart machines in education: The coming revolution in educational technology, pp. 7-35 (2001).

5. Pinkwart, N., Aleven, V., Ashley, K., \& Lynch, C.: Toward legal argument instruction with graph grammars and collaborative filtering techniques. In Proceedings of the $8^{\text {th }}$ International Conference on Intelligent Tutoring Systems, pp. 227-236 (2006). 